\title{
PROPRIEDADE FAMILIAR E DESEMPENHO EMPRESARIAL: EVIDÊNCIAS DE EMPRESAS BRASILEIRAS QUE COMPÕEM O ÍNDICE IBRX100 DA B3
}

\author{
Dalci Mendes Almeida 1 \\ Leonardo Flach 2
}

- Artigo recebido em: 17/07/2018 -" Artigo aceito em: 18/04/2020 --' Segunda versão aceita em: 25/06/2020

\section{RESUMO}

Este estudo tem por objetivo verificar se há uma relação significativa entre propriedade familiar e o desempenho de empresas brasileiras que compõem o índice IBRX100 da B3. Nesta pesquisa, a amostra é formada por 80 empresas, composta por 45 não familiares e 35 familiares. Os dados foram coletados na base de dados Economáticaß, e também no formulário de referência, para os períodos de 2012 a 2016. O modelo aplicado seguiu o estudo de Shyu (2011) e o método estatístico utilizado foi a modelagem de equações simultâneas em painel e análise descritiva. Não foram confirmadas as hipóteses de que existe relação significativa da propriedade familiar no desempenho de empresas brasileiras, e de que existe um grau de propriedade propício para otimizar o desempenho de empresas familiares brasileiras. A pesquisa traz contribuições teórica, pois avança na temática entre empresa familiar e desempenho empresarial, quando relaciona propriedade familiar com o desempenho econômico, financeiro e de mercado, e prática, por meio da evidência à gestores que o negócio com propriedade familiar nem sempre possui relação significativa nos resultados. Embora os resultados contradigam alguns estudos empíricos anteriores, o conjunto de evidências empíricas desta pesquisa devem ser interpretados com cautela, pois não há consenso entre propriedade familiar e desempenho empresarial. Conclui-se que a propriedade familiar não possui influência no desempenho empresarial.

Palavras-Chave: Propriedade familiar. Desempenho empresarial. Empresa familiar. Empresa não familiar.

\footnotetext{
1 Doutoranda do Programa de Pós-Graduação em Contabilidade da Universidade Federal de Santa Catarina (UFSC). Professora da Universidade do Sul de Santa Catarina/UNISUL. Endereço: Campus Reitor João David Ferreira Lima, s/n, Bairro Trindade, Florianópolis-SC, Brasil, CEP 88040-970. E-mail: dalci.almeida@unisul.br https://orcid.org/0000-0001-8967-0702

2 Doutor em Administração pela Universidade Federal do Rio Grande do Sul/UFRGS. Professor Associado da Universidade Federal de Santa Catarina (UFSC). Endereço: Campus Reitor João David Ferreira Lima, s/n, Bairro Trindade, Florianópolis-SC, Brasil, CEP 88040-970. E-mail: leonardo.flach@ufsc.br https://orcid.org/0000-0002-4316-0704
}

Editora responsável pela aprovação do artigo: $\mathrm{Dr}^{a}$. Bruna Camargos Avelino Editora responsável pela edição do artigo: Drª Bruna Camargos Avelino 


\title{
FAMILY PROPERTY AND ENTREPRENEURIAL PERFORMANCE: EVIDENCE OF BRAZILIAN COMPANIES COMPOSING B3 IBRX100 INDEX
}

\begin{abstract}
This study aims to verify whether there is a significant relationship between family ownership and the performance of Brazilian companies that make up the B3 IBRX100 index. In this research, the sample is formed by 80 companies, composed of 45 non-family members and 35 family members. The data were collected in the Economática® database, and also in the reference form, for the periods from 2012 to 2016. The model applied followed the study by Shyu (2011) and the statistical method used was the modeling of simultaneous panel equations. and descriptive analysis. The hypothesis that there is a significant relationship between family ownership in the performance of Brazilian companies and that there is a degree of ownership that is conducive to optimizing the performance of Brazilian family companies has not been confirmed. The research brings theoretical contributions, as it advances the theme between family business and business performance, when it relates family ownership to economic, financial and market performance, and practice, through evidence to managers that family owned business does not always have an impact results. Although the results contradict some previous empirical studies, the set of empirical evidence from this research should be interpreted with caution, as there is no consensus between family ownership and business performance. It is concluded that family ownership has no influence on business performance.
\end{abstract}

Keywords: Family property. Business performance. Family business. Non-family business.

\section{INTRODUÇÃO}

As empresas familiares possuem perspectivas, buscam viabilizar e assegurar a saúde financeira da empresa em longo prazo, possuem aversão ao risco e procuram não comprometer seus recursos em investimentos arriscados (Anderson; Duru \& Reeb, 2012). E a propriedade familiar cria uma atmosfera de compromisso necessária para um melhor desempenho (Abor \& Biekpe, 2007). Filatotchev, Lien e Piesse (2005) mencionam que a presença de proprietários de empresas de controle familiar está associada a um melhor crescimento e maior valor da empresa. Em comparação com outros acionistas, os membros da família possuem mais informações internas e podem prever mais facilmente as perspectivas de uma dada empresa (Shyu, 2011).

As empresas familiares ou que possuem gestão familiar apresentam-se menos endividadas do que as outras (Segura \& Formigoni, 2014). Shyu (2011) discorre que a propriedade familiar influencia positivamente o desempenho da firma. Barontini e Caprio (2006) argumentam que a família não prejudica o desempenho de controle familiar. Martínez, Stöhr e Quiroga (2007) constataram que empresas de controle familiar tiveram um desempenho melhor do que empresas não familiares. Anderson e Reeb (2003) consideram rentabilidade e mensuração baseadas no mercado, e que empresas familiares têm melhor desempenho do que as empresas não-familiares. James (1999) menciona que 
empresas familiares têm melhores retornos, pois possuem uma visão de longo prazo em investimentos.

Shyu (2011) destaca que ainda há desafios para os pesquisadores em verificar um consenso sobre a performance de uma empresa familiar, de ser considerada um modelo de negócio eficaz. Para Segura e Formigoni (2014, p. 56), "os estudos sobre as empresas familiares são diversos e controversos. Ora eles tratam o controle familiar, ora apenas a descrição de empresa familiar. Em outras vezes, tratam sobre a família na gestão ou nas ações da empresa". Os resultados obtidos nas pesquisas entre propriedade familiar e desempenho são diversos, dificultando a compreensão (Wang \& Shailer, 2017). Desse modo, a questão problema desse estudo consiste na seguinte: Qual a relação entre propriedade familiar e o desempenho de empresas brasileiras que compõem o índice IBRx 100 da B3?

Percebe-se nas pesquisas nacionais e internacionais (Segura \& Formigoni, 2014, Shyu, 2011; Barontini \& Caprio, 2006, Martínez, Stöhr \& Quiroga, 2007; Anderson \& Reeb, 2003; James, 1999; Lunardi, Barbosa, Rodrigues Junior, Silva \& Nakamura, 2017; Scarpin, Almeida \& Machado, 2012, Faccio, Lang \& Young, 2001, Holderness \& Sheehan, 1988), que ainda não há um consenso nos resultados obtidos sobre a propriedade familiar e desempenho. Algumas pesquisas evidenciam que há influência positiva da propriedade familiar no desempenho da empresa e outras não. Permanecendo esta dúvida, surge uma lacuna que motiva o presente estudo e a continuidade de pesquisas nesta temática.

Para Shyu (2011), a questão de saber se as empresas familiares possuem um melhor desempenho em comparação com as empresas não-familiares exige uma investigação mais aprofundada. Isto ocorre em função da diversidade dos resultados das pesquisas que têm por objetivo investigar empresas familiares e desempenho. Wang e Shailer (2017) mencionam que a propriedade familiar é uma forma organizacional importante de economia em todo o mundo. No entanto, as expectativas conflitantes dos efeitos do desempenho financeiro da propriedade familiar e os resultados empíricos aparentemente diversos constituem uma barreira à consolidação do conhecimento e ao desenvolvimento da pesquisa nesta área.

O objetivo deste estudo consiste em verificar se há uma relação significativa entre propriedade familiar e o desempenho de empresas brasileiras que compõem o índice IBRX100 da B3. Para tanto, esta pesquisa segue o mesmo modelo e método do estudo de Shyu (2011), que verificou a influência da propriedade familiar no desempenho de empresas tailandesas. Desta forma, será possível a comparação dos achados.

A avaliação de desempenho empresarial pode ser realizada por meio de índices que permitem avaliar, sob um olhar quantitativo, o desempenho econômico e financeiro das empresas. Beaver (1966) menciona que os índices permitem revelar a situação financeira negativa, de forma antecipada e estimar falência em organizações. Altman (1971) também destaca a importância da utilização dos índices para analisar a situação das empresas, pois possibilitam antever uma situação de falência.

As empresas familiares são muito comuns no Brasil e no mundo, estão presentes em diversos setores, são pequenos ou grandes negócios. De acordo com Family Firm Institute (2017), cerca de 70\% a 90\% do PIB global anualmente é 
Propriedade Familiar e Desempenho Empresarial: Evidências de Empresas Brasileiras que Compõem o Índice IBRX100 da B3

criado por empresas familiares. No Brasil, estima-se que $90 \%$ do setor privado seja formado por empresas familiares. Estas empresas são responsáveis por $50 \%$ do PIB e $85 \%$ da geração de empregos.

Para a Price Waterhouse Coopers [PWC] (2016), apesar das condições econômicas difíceis que o Brasil tem enfrentado, o segmento das empresas familiares continua vibrante e ambicioso. Colarossi, Giorgino, Steri e Viviani, (2008) mencionam que as empresas familiares representam negócios dominantes em âmbito mundial. As empresas familiares representam aproximadamente $90 \%$ de todas as formas de negócio ao redor do mundo (Acquaah, 2013).

Em pesquisas nacionais que relacionaram empresas familiares e desempenho, Lunardi et al., (2017) analisaram a criação de valor no desempenho econômico de empresas familiares e não familiares brasileiras. Tres, Serra e Ferreira (2014) verificaram a relação entre o tempo de mandato do CEO e o desempenho de empresas listadas na B3, moderado pela governança corporativa, entre 1997 e 2012. Segura e Formigoni (2014) investigaram a influência da família no endividamento das empresas abertas brasileiras.

Scarpin et al., (2012), analisaram se há diferença entre o endividamento e a lucratividade em empresas familiares e não familiares brasileiras, listadas na B3, que compõem o Índice Brasil-100. Oro, Beuren e Hein (2009) investigaram a eficiência, relacionando a estrutura de capital e o lucro operacional das empresas familiares que integram a lista das 500 maiores empresas do Brasil, conforme a revista Exame - "Melhores e Maiores de 2005. Oro, Beuren e Hein (2009a) verificaram a relação entre a estrutura de capital e o lucro operacional nas diversas gerações de empresas familiares brasileiras.

Este estudo diferencia-se, pois analisa a relação entre propriedade familiar e desempenho de empresas familiares e não familiares. Ao mesmo tempo, ele aborda a questão da endogeneidade e identifica os níveis ideais de propriedade familiar, utilizando o modelo proposto por Shyu (2011), o que ainda é pouco explorado. A contribuição teórica dessa pesquisa está no sentido de evidenciar se de fato a propriedade familiar influencia no desempenho da empresa, bem como o nível ideal de propriedade, gerando resultados que contribuam para o avanço no consenso da relação entre propriedade familiar e desempenho.

A contribuição prática da pesquisa consiste em conhecer o desempenho das empresas familiares brasileiras por meio de indicadores financeiros, econômicos e de mercado em comparação às empresas não familiares, bem como o nível adequado de propriedade familiar, para evitar a ocorrência de um desempenho desfavorável. Os resultados alcançados poderão ser utilizados para aprimorar a gestão de negócios familiares, esses que no Brasil e no mundo possuem grande representatividade no desenvolvimento econômico.

\section{PROPRIEDADE FAMILIAR E DESEMPENHO EMPRESARIAL}

A propriedade familiar apresenta custos e benefícios. Martínez et al. (2007), destacam que estudos apontam dois principais custos, o primeiro como o potencial de expropriação de recursos provenientes de outras partes interessadas na empresa e o segundo a nomeação de membros da família que não possuem competência para assumirem cargos de gerência. Já em relação aos benefícios, 
destacam-se o comprometimento a longo prazo, preservação da empresa e capacidade de monitorar os gestores.

Percebe-se que os custos e benefícios característicos de negócios familiares podem influenciar no desempenho da empresa. Anderson e Reeb (2003), com base nos resultados da sua pesquisa apoiam que os benefícios fornecidos pela propriedade familiar são superiores aos custos. Martínez et al., (2007), relatam que nas últimas décadas a influência da propriedade familiar sobre o desempenho da empresa tem sido um tema controverso entre os economistas e estudiosos de negócios, alguns resultados de estudos indicam que a propriedade familiar leva a um maior desempenho, e outros não.

Na pesquisa de Goes, Martins e Machado Filho (2017), os resultados alcançados demonstraram diferenças para resultados financeiros de empresas familiares e não familiares. Para o passivo oneroso, a presença de atributos familiares contribuiu para uma menor recorrência do uso de capitais de terceiros. Já para o lucro operacional, o resultado das empresas com atributos familiares foi inferior ao das empresas não familiares. No estudo de Lunardi et al. (2017), os resultados em determinados períodos apontaram que empresas familiares apresentam score de eficiência em relação às não familiares. Em outros períodos, as não familiares apresentaram uma melhor eficiência. Scarpin et al. (2012) não confirmaram a associação entre a gestão familiar, o endividamento e a lucratividade, com base no modelo proposto e na amostra utilizada. Faccio et al. (2001), descobriram que empresas familiares geridas por não-familiares apresentam desempenho pior do que quando são geridos por membros da família. Holderness e Sheehan (1988) mostram que os valores do $Q$ de Tobin de empresas familiares são mais baixos, ao comparar com os valores em empresas não-familiares.

Anderson e Reeb (2003) investigaram o efeito da propriedade familiar no desempenho de 403 empresas do período de 1992 a 1999, utilizando o índice Standard \& Poors das 500 maiores empresas. Os resultados indicaram que as empresas familiares superaram as não familiares significativamente, destruindo dois argumentos: primeiro, que propriedade familiar e participação geram influências negativas no desempenho de uma empresa e, segundo, que os acionistas minoritários são afetados negativamente pela propriedade familiar.

Martínez et al. (2007) estudaram o impacto da propriedade da família no desempenho de empresas familiares e não familiares chilenas. A amostra da pesquisa foi formada por 175 empresas negociadas na bolsa de valores, no período de 1995 a 2004, os resultados apontaram que o grupo de 100 empresas de controle familiar tiveram um desempenho significativamente melhor do que o grupo de 75 empresas não familiares.

Shyu (2011) analisou a influência da propriedade familiar no desempenho, identificando o percentual de propriedade familiar que maximiza o desempenho de uma empresa. A amostra foi formada por 465 empresas tailandesas registradas na bolsa de valores no período de 2002 a 2006. Os achados apontam que a propriedade familiar influencia positivamente no desempenho da empresa. Entretanto, os resultados sugerem que o excesso de participações da família pode não ser necessariamente saudável para uma empresa, indicando que a percentagem de propriedade familiar não deve exceder a 30\%, para evitar a ocorrência de um desempenho desfavorável. 
Propriedade Familiar e Desempenho Empresarial: Evidências de Empresas Brasileiras que Compõem o Índice IBRX100 da B3

Audretsch, Hülsbeck e Lehmann (2013) investigaram se a propriedade e gestão familiar levam a um melhor desempenho. A pesquisa utilizou um conjunto de dados de 386 empresas alemãs, bem como sub-amostras (quartis) distintos para as empresas familiares e pequenas e médias empresas. Os achados apontaram que o monitoramento da família pode ser o elo que faltava no debate que envolve propriedade, controle e desempenho em empresas familiares.

Castro e Aguilera (2014) estudaram a relação entre o envolvimento da família no negócio e desempenho financeiro das empresas. A amostra nacional foi formada com 6.592 empresas. Os achados apontaram que o impacto do envolvimento da família no negócio não é o produto dos componentes do envolvimento familiar isoladamente, mas que é objeto de importantes complementaridades e efeitos de substituição.

Minichilli, Brogi e Calabrò (2015) investigaram o desempenho financeiro das empresas de controle familiar em condições de "estado estacionário", em oposição a situações de carência econômica grave, bem como as condições que permitem alguns arranjos de governança para um melhor desempenho durante uma recessão econômica. A amostra foi formada por indústrias italianas familiares e não-familiares registradas na bolsa de valores no período de 2002 a 2012. Os resultados apontaram melhor desempenho das empresas controladas por familiares durante a crise financeira e econômica. Em seguida, com foco estritamente em empresas familiares, descobriu-se que as configurações mistas (CEOs família com menor concentração de propriedade familiar) produzem um melhor desempenho em face a perigos externos.

Trasobares e Górriz (2016) estudaram o impacto individual e conjunto de controle familiar e diversificação/criação de relação de valor sobre o desempenho, considerando a natureza do último proprietário de grupos não familiares. A amostra foi formada por 99 empresas controladoras e subsidiárias espanholas, cotadas na bolsa de valores, no período de 2000 a 2005 . Os resultados indicaram como o controle familiar tem um impacto negativo sobre o valor $Q$ de Tobin, e que as diferenças são maiores entre os grupos familiares e não familiares controlados pelos bancos e/ou agentes estrangeiros. Demonstraram também como a diversificação não afeta a criação de valor individualmente ou considerando o possível efeito moderador de propriedade familiar.

Wang e Shailer (2017) avaliaram se existe uma relação subjacente entre a propriedade familiar e o desempenho financeiro da empresa. A amostra foi formada por 43 estudos empíricos de empresas listadas em 17 mercados emergentes. Os achados da pesquisa apontaram uma relação global positiva entre a concentração da propriedade familiar e o desempenho da empresa, bem como a relação de propriedade e desempenho familiar é maior nos mercados emergentes com níveis de qualidade institucional relativamente maiores.

Infere-se do exposto que a propriedade familiar pode possuir uma relação significativa com o desempenho empresarial. Neste sentido, e a partir dos relatos de pesquisas anteriormente expostas, apresenta-se a primeira hipótese da pesquisa:

$H_{1}$ : Há uma relação significativa entre propriedade familiar e o desempenho de empresas brasileiras. 
São fornecidos aos membros da família incentivos consideráveis para supervisionar as funções de gestores, com o objetivo de melhorar o desempenho empresarial. Além disso, uma expansão da propriedade familiar ajuda a evitar o problema da agência. Desse modo, o grau de propriedade familiar leva a alterações no desempenho da empresa (Shyu, 2011). Wang e Shailer (2017), com base nos resultados de sua pesquisa concordam com a visão de que a apropriação familiar concentrada, pode monitorar melhor os gerentes ou melhor alinhar a maioria e os interesses dos acionistas minoritários, para melhorar o valor corporativo nos mercados emergentes.

Anderson e Reeb (2003) utilizaram o ROA e Q de Tobin como variáveis proxy para o desempenho, os resultados apontam que o desempenho inicial de empresas familiares nos Estados Unidos melhora juntamente com uma expansão na propriedade familiar. O desempenho da firma atinge seu pico quando a propriedade familiar é de 30,8\% (medido usando o EBITDA), 27,6\% (medido usando Lucro Líquido LL), e 31\% (medido com o uso da taxa Tobin Q). No entanto, quando a propriedade familiar continua a aumentar, o desempenho da firma começa a piorar.

Barontini e Caprio (2006), mencionam que o achado de que propriedade familiar é determinado endogenamente implica que os investigadores devem considerar a endogeneidade quando determinar as relações entre propriedade familiar e desempenho de uma empresa. Trasobares e Górriz (2016) em sua pesquisa utilizam a correção de duas etapas de Heckman para remover o viés de seleção e a endogeneidade de propriedade da família. Wang e Shailer (2017), também estudaram a relação entre a concentração de propriedade familiar e desempenho para empresas listadas em mercados emergentes, encontram que uma quantidade significativa de diferenças nas relações entre os estudos é influenciada pelas escolhas dos pesquisadores sobre os métodos de regressão para avaliar a endogeneidade.

Infere-se do exposto que grau de propriedade pode aumentar ou diminuir de acordo com o desempenho da empresa. Desse modo, e a partir dos relatos de pesquisas anteriormente expostas, apresenta-se a segunda hipótese da pesquisa:

$\mathrm{H}_{2}$ : Há uma relação significativa entre o desempenho de empresas familiares brasileiras e o seu grau de propriedade.

\section{PROCEDIMENTOS METODOLÓGICOS}

Em relação ao delineamento a pesquisa classifica-se quanto aos objetivos como descritiva, em relação à abordagem do problema quantitativa e no que se refere aos procedimentos, como documental.

\subsection{Seleção da Amostra}

A população da pesquisa é formada pelas empresas listadas no índice IBRx100 da B3, familiares e não familiares que são as empresas com maior movimentação na bolsa, compreendendo o período de 2012 a 2016. Foram excluídas da amostra as empresas que possuem estrutura financeira diferenciada como instituições financeiras e seguradoras, constituindo a amostra de 80 
empresas representando, $80 \%$ da população, composta por 35 empresas familiares e 45 não familiares. Esses critérios levam à formação de um painel desbalanceado de 400 observações.

Shyu (2011, p. 401), considerou empresa familiar a empresa que preenche ao menos uma das duas condições seguintes: a) total de propriedade familiar, superior a dez por cento, com membros da família como membros do conselho de administração; ou b) mais da metade dos assentos no conselho detidos pelos membros da família. Considerou-se empresa familiar nesta pesquisa, empresas com propriedade familiar superior a 10\% conforme Shyu (2011) e Anderson e Reeb (2003).

Os dados relacionados a propriedade das empresas familiares e propriedade institucional foram coletados no formulário de referência. A propriedade familiar no item assembleia e administração em relações familiares, composição e experiência profissional da administração e do conselho fiscal e no item controle e grupo econômico em posição acionária e a propriedade institucional no item controle e grupo econômico em posição acionária. As variáveis de desempenho foram coletadas a partir da base de dados Economática®.

\subsection{Procedimentos de Análise dos Dados}

Para Shyu (2011), uma equação simultânea pode ser estimada para o sistema de propriedade familiar e do desempenho, utilizando o método de mínimos quadrados em dois estágios 2SLS para resolver o problema da endogeneidade potencial. Para Wooldridge (2011, p. 531), os "modelos de equações simultâneas são apropriados quando cada equação no sistema tem uma interpretação ceteris paribus." O sistema de equação simultânea pode ser expresso da seguinte forma:

Desempenho $_{i t}=\beta_{1 i}+\beta_{2}$ propriedade familiar $_{i t}+\beta_{3}$ (propriedade familiar $) 2_{i t}$

$+\beta_{4}$ tamanho da empresa ${ }_{i t}+\beta_{5}$ proporção da dívida de longo prazoit

$+\beta_{6}$ rendimento do dividendo it $+\beta_{7}$ propriedade institucionalit

$+\beta_{8}$ Intangível/vendasit $+e_{i t}$

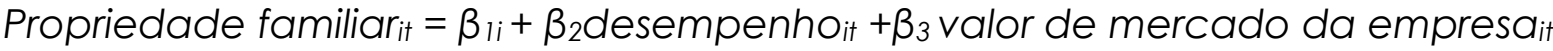

$+\beta_{4}$ proporção da dívida de longo prazoit

$+\beta_{5}$ rendimento do dividendoit

$+\beta_{6}$ volatividade do retorno it $+e_{i t}$

Para medir o desempenho da empresa, serão utilizados os indicadores de rentabilidade (ROA) e o indicador de avaliação (Q de Tobin). A taxa ROA é calculada de duas formas:

1) Lucro líquido dividido pelo valor contábil dos ativos totais, denominadas ROA $(\mathrm{LL})=\mathrm{LL} / \mathrm{AT}$. 
2) $O$ EBITDA dividido pelo valor contábil dos ativos totais, denominado como ROA $($ EBITDA $)=$ EBITDA $/ A T$

Sabe-se que os indicadores ROA (LL) e ROA (EBITDA) podem ser distorcidos pelos tratamentos contábeis, com isso um indicador de mercado $Q$ de Tobin será considerado. Shyu (2011, p. 403), menciona que o "Q de Tobin considera o custo do capital, riscos e valor do dinheiro no tempo e evita problemas associados com os princípios contábeis". $O Q$ de Tobin será calculado de forma aproximada. Chung e Pruitt (1994) explicam que o "VMA" consiste no valor de mercado da empresa e o "D" é composto pelo valor contábil dos passivos circulantes, somado do valor contábil dos passivos não circulantes e o valor contábil dos estoques e deste valor subtraído do valor contábil dos ativos circulantes. O resultado obtido "VMA" somado ao "D "é, então, dividido pelo valor contábil do ativo total. $Q$ de Tobin = (VMA + D) $/$ AT.

A propriedade familiar será calculada considerando a razão entre o número de ações de todas as classes em poder de membros da família e o total de ações em circulação.

Na análise de regressão em dados em painel se utilizará como variáveis de controle as que são apresentadas na Figura 1: 
Propriedade Familiar e Desempenho Empresarial: Evidências de Empresas Brasileiras que Compõem o Índice IBRX100 da B3

\begin{tabular}{|c|c|c|c|c|}
\hline Variáveis & $\begin{array}{c}\text { Siglas/ } \\
\text { Fórmulas }\end{array}$ & Descrição & $\begin{array}{c}\text { Sinal } \\
\text { Esperado }\end{array}$ & Autores \\
\hline $\begin{array}{l}\text { Tamanho da } \\
\text { empresa }\end{array}$ & LnAT & $\begin{array}{l}\text { Logarítmo natural dos } \\
\text { ativos totais }\end{array}$ & + & $\begin{array}{l}\text { Andres (2008); } \\
\text { Shyu (2011) }\end{array}$ \\
\hline $\begin{array}{l}\text { Proporção da } \\
\text { dívida de longo } \\
\text { prazo }\end{array}$ & DLP/AT & $\begin{array}{l}\text { Dívidas de longo prazo } \\
\text { dividido pelo ativo } \\
\text { total. }\end{array}$ & - & $\begin{array}{l}\text { Chen et al. } \\
\text { (2003); Singh e } \\
\text { Davidso (2003); } \\
\text { Demsetz e } \\
\text { Villalonga (2001); } \\
\text { Jensen et al. } \\
\text { (1992); Shyu } \\
\text { (2011) }\end{array}$ \\
\hline $\begin{array}{l}\text { Rendimento do } \\
\text { Dividendo }\end{array}$ & DPA/PMA & $\begin{array}{l}\text { Dividendo por ação } \\
\text { divido pelo preço de } \\
\text { mercado da ação }\end{array}$ & + & $\begin{array}{l}\text { Jensen et al. } \\
\text { (1992); Chen e } \\
\text { Steiner (1999); } \\
\text { Ross (1977) }\end{array}$ \\
\hline $\begin{array}{l}\text { Propriedade } \\
\text { institucional }\end{array}$ & TP/NAC & $\begin{array}{l}\text { Total de participações } \\
\text { de agências } \\
\text { governamentais, } \\
\text { instituições financeiras } \\
\text { e investidores } \\
\text { institucionais } \\
\text { estrangeiros dividido } \\
\text { pelo número de ações } \\
\text { em circulação }\end{array}$ & + & $\begin{array}{l}\text { Chen et al. } \\
\text { (2003); Pound } \\
\text { (1991); Black } \\
\text { (1992); Shyu } \\
\text { (2011) }\end{array}$ \\
\hline $\begin{array}{l}\text { Intangível sobre } \\
\text { as vendas }\end{array}$ & $\begin{array}{l}\text { Intangível } \\
\text { /Vendas }\end{array}$ & $\begin{array}{l}\text { Intangível sobre as } \\
\text { vendas }\end{array}$ & + & $\begin{array}{l}\text { Morck, Shleifer, } \\
\text { Vishny (1988); } \\
\text { McConnell e } \\
\text { Servaes (1990); } \\
\text { Shyu (2011) }\end{array}$ \\
\hline $\begin{array}{l}\text { Valor de } \\
\text { mercado }\end{array}$ & LVM & $\begin{array}{l}\text { Logaritmo do valor de } \\
\text { mercado }\end{array}$ & + & $\begin{array}{l}\text { Casson (1999); } \\
\text { Shyu (2011) }\end{array}$ \\
\hline $\begin{array}{l}\text { Volatilidade de } \\
\text { retorno }\end{array}$ & VR & $\begin{array}{l}\text { Desvio padrão do } \\
\text { histórico anual de } \\
\text { retornos diários }\end{array}$ & - & $\begin{array}{l}\text { Chen et al. } \\
\text { (2003); Shyu } \\
(2011)\end{array}$ \\
\hline
\end{tabular}

Figura 1 - Variáveis de controle

Fonte: Adaptado de Shyu (2011)

Para a variável tamanho da empresa, espera-se que tenha uma relação positiva com o desempenho (Andres, 2008). Para Shyu (2011), as empresas maiores são mais suscetíveis de serem mais eficientes na utilização de ativos e se beneficiar de economias de escala. Espera-se uma correlação negativa da proporção da dívida de longo prazo com o desempenho da empresa, pois a sua utilização aumenta os custos de falência (Chen, Guo \& Mande, 2003; Singh \& Davidson, 2003; Demsetz \& Villalonga, 2001). Além disso, espera-se que essa variável apresente uma associação negativa com a propriedade familiar, pois as empresas com dívidas elevadas, estão sujeitas a um maior monitoramento no mercado externo, porém menos controlo interno é imposto sobre estas empresas (Chen et al., 2003, Guo \& Mande, 2003; Jensen, Solberg \& Zorn, 1992).

$O$ rendimento do dividendo pode ser positivamente relacionado ao desempenho de uma empresa, pois pode diminuir o custo de agência (Jensen et al., 1992; Chen \& Steiner, 1999). Quando a empresa não possui boas oportunidades de investimento, pode ser negativamente relacionado ao 
desempenho de uma empresa (Ross, 1977). A propriedade institucional atua como um monitor externo e está prevista para ser associada positivamente com o desempenho (Chen et al., 2003; Pound, 1991; Black, 1992).

A variável Intangível/Vendas representa a importância dos intangíveis para vendas e funciona como um controle para a especificidade de ativos (Shyu, 2011 ). Pode influenciar positivamente o desempenho, como seria de esperar de ativos incorpóreos para melhorar o desempenho (Morck et al., 1988; McConnell \& Servaes, 1990). Espera-se que o valor de mercado da empresa seja positivamente relacionado com a propriedade familiar (Casson, 1999). De acordo com (Shyu, 2011, p. 404), "as empresas com alta valorização e o potencial de crescimento futuro pode inspirar os membros da família para aumentar as suas participações". A volatilidade de retorno representa o risco operacional, os gerentes em empresas com alto riscos operacionais requerem níveis elevados de propriedade com o objetivo de ganhar reconhecimento do mercado (Chen et al., 2003).

Como essa pesquisa segue o modelo proposto por Shyu (2011) será utilizado um conjunto de dados em painel abrangendo o período de cinco anos para examinar transversais de variações nas relações entre propriedade familiar e desempenho de uma empresa. Será analisado também os efeitos de potenciais vieses decorrentes da endogeneidade de propriedade e desempenho. Para Wooldridge (2011, p. 531), "aplicações de equações simultâneas com dados em painel são muito poderosas, já que nos possibilitam controlar a heterogeneidade não observada ao mesmo tempo em que estamos lidando com a simultaneidade".

\section{ANÁLISE E DESCRIÇÃO DOS DADOS}

\subsection{Análise dos Dados}

Na Tabela 1 são apresentadas as estatísticas descritivas das variáveis, para as empresas incluídas na amostra.

Tabela 1

Estatísticas descritivas das variáveis

\begin{tabular}{l|c|c|c|c}
\multicolumn{1}{c|}{ Variáveis } & Mínimo & Máximo & Média & $\begin{array}{c}\text { Desvio } \\
\text { Padrão }\end{array}$ \\
\hline Q de Tobin & 0,07 & 52,84 & 1,42 & 2,82 \\
ROA (EBITDA) (\%) & $-25,55$ & 34,46 & 9,80 & 7,66 \\
ROA (LL) (\%) & $-43,02$ & 32,74 & 4,60 & 7,38 \\
Propriedade Familiar (\%) & 0,00 & 74,05 & 36,42 & 18,04 \\
Intangível sobre as Vendas (\%) & 0,00 & 316,17 & 41,38 & 56,74 \\
Proporção da dívida de LP (\%) & 0,00 & 88,24 & 34,24 & 16,53 \\
Volatilidade do Retorno (\%) & 0,00 & 7,00 & 2,29 & 0,92 \\
Tamanho da empresa (Mil R\$) & 92 & $900.135,0$ & $33.036,0$ & $95.576,0$ \\
Valor de mercado da empresa & 386 & $279.979,0$ & $17.404,0$ & $40.138,0$ \\
(Mil R\$) & 0,00 & 118,83 & 4,00 & 7,92 \\
Rendimento do Dividendo (\%) & 0,00 & 97,65 & 23,66 & 21,43 \\
Propriedade Institucional (\%) & & & & \\
\hline
\end{tabular}

Fonte: Elaborada pelos autores. 
Percebe-se na Tabela 1, que para os indicadores de desempenho de valor de mercado, o valor máximo de $Q$ de Tobin é 52,84, o valor mínimo é 0,07 e a média de 1,42, ou seja, uma diferença considerável entre o mínimo é o máximo, dado $\circ Q$ de Tobin de uma única empresa ter sido muito elevado. Para os indicadores de desempenho contábil, o valor máximo de ROA (EBITDA) é de $34,46 \%$, o valor mínimo é $-25,55 \%$ e a média $7,66 \%$. O valor máximo de ROA (LL) é de $32,74 \%$, o valor mínimo é de $-43,02 \%$ e a média $4,60 \%$. Percebe-se variações significativas entre os indicadores de desempenho de mercado e contábil das empresas analisadas, coadunando com os resultados da pesquisa de Shyu (2011) que apresentaram variações significativas entre os indicadores de desempenho das empresas incluídas na amostra. As variáveis intangível sobre as vendas, tamanho da empresa, valor de mercado da empresa, também tiveram grandes oscilações, apresentando uma média respectivamente de $41,38 \%$, R $\$ 33.036$ e R\$ 17.404 respectivamente. Na pesquisa de Shyu (2011) o indicador pesquisa e desenvolvimento sobre as vendas também teve grande oscilação, o mesmo não aconteceu com as variáveis tamanho da empresa e valor de mercado. A propriedade familiar e propriedade institucional são as que apresentam variações significativas.

Em seguida, aplicou-se o teste t de Student no intuito de comparar as médias de duas amostras (grupos) independentes, com o objetivo de avaliar se elas são oriundas de populações com médias iguais ou diferentes, neste caso as médias entre empresas familiares e não familiares. O teste t de Student foi proposto em 1908 por William Sealy Gosset, e consiste em um importante teste de hipóteses que pode ser aplicado para amostras com distribuição paramétrica, que foi o caso da presente pesquisa. As médias obtidas de amostras $(\bar{x})$ são estimativas de parâmetros populacionais ( $\mu$ ) que são desconhecidos; além disso, estão sujeitas a variação em decorrência do efeito do acaso na seleção dos sujeitos. Portanto, pode-se inferir sobre a diferença entre médias populacionais com base em médias de duas amostras, entre empresas familiares e não familiares. Na Tabela 2 constam os resultados do teste T de médias entre empresas familiares e não familiares. 
Tabela 2

Teste T de médias entre empresas familiares e não familiares

\begin{tabular}{|c|c|c|c|c|}
\hline Variáveis & $\begin{array}{c}\text { Média } \\
\text { Familiares }\end{array}$ & $\begin{array}{l}\text { Média Não } \\
\text { Familiares }\end{array}$ & Teste $t$ & Sig. \\
\hline Q de Tobin & 131,33 & 150,50 & $\begin{array}{l}-0,661 \\
-0,727\end{array}$ & $\begin{array}{l}0,509 \\
0,468\end{array}$ \\
\hline ROA (EBITDA) (\%) & 10,41 & 9,32 & $\begin{array}{l}1,396 \\
1,432\end{array}$ & $\begin{array}{l}0,163 \\
0,153\end{array}$ \\
\hline ROA (LL) (\%) & 5,04 & 4,25 & $\begin{array}{l}1,047 \\
1,064\end{array}$ & $\begin{array}{l}0,296 \\
0,288\end{array}$ \\
\hline Propriedade Familiar (\%) & 36,63 & & $\begin{array}{l}30,121 \\
26,870\end{array}$ & $\begin{array}{l}0,000 \\
0,000\end{array}$ \\
\hline Intangível sobre as Vendas (\%) & 33,43 & 47,71 & $\begin{array}{l}-2,478 \\
-2,565\end{array}$ & $\begin{array}{l}0,014 \\
0,011\end{array}$ \\
\hline Proporção da dívida de LP (\%) & 36,11 & 32,76 & $\begin{array}{l}1,991 \\
1,959\end{array}$ & $\begin{array}{l}0,047 \\
0,051\end{array}$ \\
\hline Volatilidade do Retorno (\%) & 2,30 & 2,28 & $\begin{array}{l}0,151 \\
0,152\end{array}$ & $\begin{array}{l}0,880 \\
0,879\end{array}$ \\
\hline Tamanho da empresa (Mil R\$) & 14.854 & 47.448 & $\begin{array}{l}-3,385 \\
-3,784\end{array}$ & $\begin{array}{l}0,001 \\
0,000\end{array}$ \\
\hline $\begin{array}{l}\text { Valor de mercado da empresa } \\
\text { (Mil R } \$ \text { ) }\end{array}$ & 8.667 & 24.460 & $\begin{array}{l}-3,863 \\
-4,278\end{array}$ & $\begin{array}{l}0,000 \\
0,000\end{array}$ \\
\hline Rendimento do Dividendo (\%) & 2,53 & 5,21 & $\begin{array}{l}-3,340 \\
-3,637\end{array}$ & $\begin{array}{l}0,001 \\
0,000\end{array}$ \\
\hline Propriedade Institucional (\%) & 15,34 & 30,30 & $\begin{array}{l}-7,291 \\
-7,655\end{array}$ & $\begin{array}{l}0,000 \\
0,000\end{array}$ \\
\hline
\end{tabular}

Fonte: Elaborada pelos autores.

Observa-se na Tabela 2, que os indicadores $Q$ de Tobin, ROA (EBITDA) e ROA (LL), ou seja, indicadores de desempenho de mercado e contábil e a volatilidade de retorno, não possuem diferenças estatisticamente significativas ao nível de $\mathrm{P}<0,05$, entre empresas familiares e não familiares. Diferente do estudo de Shyu (2011), que observou diferenças significativas nestes indicadores, em que as empresas familiares funcionam significativamente melhor do que as empresas não familiares nas variáveis de desempenho. A volatilidade do retorno foi nas empresas familiares menor que nas empresas não familiares.

As variáveis tamanho da empresa, valor de mercado da empresa, rendimento do dividendo e propriedade institucional, são significativamente menores entre empresas familiares, coadunando com os achados de Shyu (2011). Nas variáveis denominadas intangível sobre as vendas e proporção da dívida de longo prazo, as diferenças também foram significativas, mas os achados diferem do estudo de Shyu (2011). Para Wang e Shailer (2017), os resultados obtidos nas pesquisas entre propriedade familiar e desempenho são diversos, dificultando a compreensão. Martínez et al., (2007), relatam que alguns resultados de estudos indicam que a propriedade familiar leva a um maior desempenho, e outros não.

As Tabelas 3 e 4 demonstram os resultados das equações simultâneas em painel para as empresas familiares. 
Propriedade Familiar e Desempenho Empresarial: Evidências de Empresas Brasileiras que Compõem o Índice IBRX100 da B3

Tabela 3

Equações simultâneas de propriedade familiar e desempenho método 2SLS

\begin{tabular}{l|c|c|c}
\hline Variáveis & ROA (EBITDA) & ROA (LL) & Q de Tobin \\
\hline & $0,042^{*}$ & $0,049^{*}$ & 0,221 \\
Propriedade Familiar^2 & 0,631 & 0,540 & $-5,892$ \\
Propriedade Familiar & 0,258 & 0,114 & 0,157 \\
& $-0,225$ & $-0,278$ & 4,374 \\
Tamanho da Empresa & $0.000^{* * *}$ & 0,249 & $0,000^{* * *}$ \\
Proporção da dívida de LP & $-0,0443$ & $-0,012$ & $-0,991$ \\
& 0,760 & $0,019^{* *}$ & 0,415 \\
Rendimento do Dividendo & 0,017 & $-0,117$ & 0,713 \\
& 0,640 & 0,555 & 0,145 \\
Propriedade institucional & $-0,072$ & 0,081 & $-3,508$ \\
& $0,005^{* *}$ & 0,161 & $0,030^{* *}$ \\
Intangível sobre as vendas & $-0,160$ & $-0,070$ & $-1,913$ \\
& $0,058^{*}$ & 0,174 & $0,010^{* * *}$ \\
Constante & $-0,072$ & $-0,045$ & $-1,520$ \\
Ponto de Inflexão & $0,000^{* * *}$ & $0,059^{*}$ & $0,000^{* * *}$ \\
& 0,854 & 0,335 & 17,129 \\
Hausman tes† & 17,83 & 25,79 & 37,12 \\
Chow & $0,000^{* * *}$ & $0,021^{* *}$ & $0,001^{* * *}$ \\
LM Breusch-Pagan & 29,172 & 16,457 & 24,376 \\
R2 within & $0,000^{* * *}$ & $0,000^{* * *}$ & $0,000^{* * *}$ \\
R2 between & $0,000^{* * *}$ & $0,000^{* * *}$ & $0,000^{* * *}$ \\
R2 Overall & 0,242 & 0,146 & 0,293 \\
& 0,115 & 0,463 & 0,194 \\
Notas: & 0,094 & 0,399 & 0,166 \\
\hline
\end{tabular}

Notas:

Equação: Desempenhoit $=\beta_{1 i}+\beta_{2}$ propriedade familiarit $+\beta_{3}$ (propriedade familiar) $2_{i t}$ $+\beta_{4}$ tamanho da empresait $+\beta_{5}$ proporção da dívida de longo prazoit $+\beta_{6}$ rendimento do dividendoit $+\beta$ propriedade institucionalit $+\beta_{8}$ Intangível sobre as vendasit $+e_{\text {it }}$

$*, * *, * * *$ : significativo a 10,5 e $1 \%$.

Fonte: Elaborada pelos autores.

De acordo com os testes LM de Breusch-Pagan, F de Chow e Hausman, o modelo de dados em painel com efeitos fixos é mais apropriado. Percebe-se na Tabela 03 que, quando a as variáveis dependentes forem ROA (EBITDA), ROA (LL), os coeficientes de propriedade familiar são negativos e não significativos. No entanto, quando a variável dependente for $\circ Q$ de Tobin, o coeficiente de propriedade familiar é positivo e também sem significância.

Observa-se ainda, que o tamanho da empresa está negativamente e significativamente correlacionado apenas com o ROA (EBITDA) e $Q$ de Tobin. $O$ índice de dívida de longo prazo está correlacionado negativamente e de forma significativa apenas com o ROA (LL), coadunando com os achados de Chen et al., 2003, Singh e Davidson, 2003, Demsetz e Villalonga, 2001, Jensen et al., 1992, Shyu, 2011 . O rendimento de dividendo não apresentou significância em nenhum dos períodos analisados. A propriedade institucional está correlacionada negativamente e de forma significativa com $O$ ROA (EBITDA) e $Q$ de Tobin, apenas; $O$ intangível sobre as vendas está correlacionado negativamente e de forma significativa com o ROA (EBITDA) e $Q$ de Tobin. De maneira geral, os achados não corroboram com o estudo de Shyu (2011). 
A Tabela 4 evidencia a propriedade familiar e o desempenho empresarial, considerando as variáveis de desempenho contábil e de mercado e a propriedade familiar como dependente.

\section{Tabela 4}

Propriedade familiar e desempenho empresarial

\begin{tabular}{|c|c|c|c|}
\hline Variáveis & $\begin{array}{l}\text { Propriedade } \\
\text { Familiar }\end{array}$ & $\begin{array}{l}\text { Propriedade } \\
\text { Familiar }\end{array}$ & $\begin{array}{c}\text { Propriedade } \\
\text { Familiar }\end{array}$ \\
\hline ROA (EBITDA) & $\begin{array}{c}0,059^{* *} \\
0,199\end{array}$ & & \\
\hline $\mathrm{ROA}(\mathrm{LL})$ & & $\begin{array}{c}0,473 \\
0,0943399\end{array}$ & \\
\hline O de Tobin & & & 0,605 \\
\hline & 0,362 & 0,624 & 0,646 \\
\hline Valor de Mercado & $\begin{array}{r}-0,010 \\
0,756\end{array}$ & $\begin{array}{c}-0.005 \\
0,689\end{array}$ & $\begin{array}{l}-0,005 \\
0,850\end{array}$ \\
\hline Proporção da dívida LP & $\begin{array}{l}0,020 \\
0,796\end{array}$ & $\begin{array}{l}0,027 \\
0,720\end{array}$ & $\begin{array}{l}0,012 \\
0,851\end{array}$ \\
\hline Rendimento do Dividendo & $\begin{array}{l}-0,061 \\
0,297\end{array}$ & $\begin{array}{l}-0,087 \\
0,275\end{array}$ & $\begin{array}{l}-0,045 \\
0,285\end{array}$ \\
\hline Volatilidade do retorno & $\begin{array}{l}0,008 \\
0,013\end{array}$ & $\begin{array}{c}0,008 \\
0,030^{* *}\end{array}$ & $\begin{array}{c}0,008 \\
0,033^{* *}\end{array}$ \\
\hline Constante & $\begin{array}{l}0,480 \\
0,946\end{array}$ & $\begin{array}{l}0,419 \\
0,786\end{array}$ & $\begin{array}{l}0,421 \\
0,911\end{array}$ \\
\hline Hausman test & 1,184 & 2,435 & 1,516 \\
\hline Chow & $0,000^{* * *}$ & $0,000 * * *$ & $0,000^{* * *}$ \\
\hline LM Breusch-Pagan & $0,000^{* * *}$ & $0,000^{* * *}$ & $0,000^{* * *}$ \\
\hline $\mathrm{R}^{2}$ within & 0,040 & 0,017 & 0,018 \\
\hline$R^{2}$ between & 0,004 & 0,009 & 0,000 \\
\hline $\mathrm{R}^{2}$ overall & 0,002 & 0,005 & 0,000 \\
\hline
\end{tabular}

Notas:

Equação: Propriedade familiarit $=\beta 1 i+\beta 2$ desempenhoit $+\beta 3$ valor de mercado da empresait $+\beta 4$ proporção da dívida de longo prazoit + $\beta 5$ rendimento do dividendoit + $\beta 6$ volatividade do retornoit +eit

$*, * *, * *$ : significativo a 10,5 e $1 \%$.

Fonte: Elaborada pelos autores.

De acordo com os testes LM de Breusch-Pagan, F de Chow e Hausman, o modelo de dados em painel com efeitos aleatórios é o mais apropriado. Percebese na Tabela 04 uma correlação negativa e não significativa entre o valor de mercado e a propriedade familiar, o que corroborou em parte com o estudo de Shyu (2011) que identificou evidência fraca de um efeito positivo do valor de mercado na propriedade familiar, mesmo assim Shyu (2011), destaca que as empresas com alta valorização e o potencial de crescimento futuro podem inspirar os membros da família para aumentar as suas participações.

No que se refere a proporção da dívida de longo prazo, os achados apontam uma relação positiva e insignificativa com a propriedade familiar, diferente do resultado de Shyu (2011), que encontrou uma correlação negativa entre a proporção da dívida de longo prazo e a propriedade familiar, sugerindo que menos monitoramento interno ocorre em empresas familiares. Para a variável rendimento do dividendo, a correlação também foi negativa e insignificante em 
Propriedade Familiar e Desempenho Empresarial: Evidências de Empresas Brasileiras que Compõem o Índice IBRX100 da B3

todos os indicadores analisados, corroborando com o estudo de Shyu (2011), em que o rendimento de dividendos não teve efeito sobre a propriedade familiar. Na volatilidade do retorno a correlação foi positiva, mas também insignificante, no estudo de Shyu (2011) a correlação foi positiva, indicando que a volatilidade pode aumentar o valor da propriedade familiar, pois a incerteza torna o acompanhamento das contribuições gerenciais para 0 desempenho da empresa muito mais difícil.

A Tabela 4 ainda demonstra que, quando a propriedade familiar é uma variável dependente, os coeficientes de ROA (EBITDA), ROA (LL) e Q de Tobin são todos positivos, mas apenas ROA (EBITDA) é estatisticamente significativo. Desse modo, não se pode confirmar a relação conjuntamente endógena entre a propriedade familiar e o desempenho das empresas, ou seja, não se pode afirmar que esses dois fatores se influenciam mutuamente.

\subsection{Discussão dos Resultados}

Os resultados apontam, que não podemos afirmar que a propriedade familiar melhora o desempenho e consequentemente uma maior propriedade familiar, leva a um melhor desempenho, diferentemente dos achados de Shyu (2011), que apontaram que a propriedade familiar melhora o desempenho e que uma maior propriedade familiar, leva a um melhor desempenho. Os achados da pesquisa de Wang e Shailer (2017), apontaram uma relação global positiva entre a concentração da propriedade familiar e o desempenho da empresa. Lunardi et al., (2017), Scarpin et al., (2012), Faccio et al., 2001, Holderness e Sheehan (1988), também não encontraram uma relação positiva entre propriedade familiar e desempenho empresarial. Diante de exposto, infere-se que H1: Há uma relação significativa entre propriedade familiar e o desempenho de empresas brasileiras, não pode ser confirmada.

Percebe-se ainda que, quando ROA (EBITDA) e ROA (LL) são os indicadores de desempenho, além do coeficiente de propriedade familiar ser negativo e insignificante o coeficiente de propriedade da família ao quadrado é positivo e significativo. Quando a propriedade familiar é de aproximadamente $17,83 \%$ e $25,13 \%$ por cento, ROA (EBITDA) e ROA (LL) atingem seu pico, porém considerar a propriedade familiar ideal não foi possível, dado a significância das variáveis.

Quando $O Q$ de Tobin é usado como indicador de desempenho, o desempenho da empresa exibe uma relação positiva com a propriedade familiar, porém insignificante; em relação ao quadrado da propriedade familiar a correlação é negativa e também insignificante. Portanto, determinar a propriedade familiar ideal para maximizar o valor de mercado de uma empresa também não é possível, corroborando com os achados de Shyu (2011).

Desse modo, infere-se que a H2: Há uma relação significativa entre o desempenho de empresas familiares brasileiras e o seu grau de propriedade, não pode ser confirmada.

O aumento da propriedade familiar não melhora o desempenho, contribuindo também para não confirmação da $\mathrm{Hl}$, ou seja, não existe uma relação significativa entre a propriedade familiar e o desempenho de empresas brasileiras. Segura e Formigoni (2014), Shyu (2011), Barontini e Caprio (2006), Martínez et al. (2007), Anderson e Reeb (2003), James (1999), Lunardi et al., 2017, 
Scarpin et al. (2012), Faccio et al. (2001), Holderness e Sheehan (1988) destacam que ainda não existe um consenso nos resultados obtidos no que se refere a propriedade familiar e desempenho. Para Goes et al. (2017), os estudos que versam sobre o desempenho de empresas familiares são muitas vezes dicotômicos, alguns apresentam resultados desfavoráveis ao modelo familiar e outros resultados claramente positivos.

\section{CONSIDERAÇÕES FINAIS}

A pesquisa objetivou verificar se há uma relação significativa entre propriedade familiar e o desempenho de empresas brasileiras que compõem o índice IBRx100 da B3. Os pressupostos teóricos desse estudo foram de que as empresas familiares, são mais conservadoras, possuem perspectivas e buscam viabilizar e assegurar a saúde financeira da empresa em longo prazo e apresentam aversão ao risco, podendo levar ao melhor desempenho. Para tanto, utilizou-se uma amostra de 80 empresas, sendo 45 não familiares e 35 familiares, do período de 2012 a 2016 e a técnica estatística equações simultâneas em painel, além da estatística descritiva.

As hipóteses $\mathrm{H1}$ : Há uma relação significativa entre propriedade familiar e o desempenho de empresas brasileiras e H2: Há uma relação significativa entre o desempenho de empresas familiares brasileiras e o seu grau de propriedade, não foram confirmadas.

Em relação a $\mathrm{Hl}$, os resultados apontaram que se os indicadores forem de rentabilidade ROA (LL) e ROA (EBITDA), ou de avaliação $Q$ de Tobin, não podemos afirmar que a propriedade familiar afeta positivamente o desempenho da empresa. Além disso, os achados não revelam uma relação conjuntamente endógena entre o desempenho da empresa e a propriedade familiar, ou seja, um aumento na propriedade familiar não aumenta o desempenho da empresa. Desse modo, os achados em empresas brasileiras, não corroboram com os resultados da pesquisa de Shyu (2011) realizado em empresas tailandesas, em que a relativa concentração da propriedade familiar afeta o desempenho da empresa, pois os membros da família têm mais informações internas, podem prever as perspectivas com mais facilidade e tomar boas decisões de forma que influenciam de maneira positiva o desempenho.

A propriedade das empresas familiares, caracterizada como concentrada, não possui relação significativa com o desempenho das empresas, a concentração de propriedade, no caso brasileiro, pode indicar menor desempenho das empresas familiares, o que pode justificar os resultados obtidos, diferente dos achados de Shyu (2011), em que a maioria das empresas familiares em Taiwan possui uma participação relativamente concentrada, o que pode propiciar ao melhor desempenho.

No que se refere a H2, não foi possível identificar os níveis de propriedade familiar que são mais benéficos para o desempenho das empresas, em função da correlação e significância das variáveis, diferente dos achados de Shyu (2011) que os resultados revelaram que a rentabilidade de uma empresa (ROA) aumenta inicialmente com o aumento das participações familiares e atinge seu pico quando a propriedade familiar é de aproximadamente $30 \%$. 
A pesquisa trouxe implicações teóricas e práticas. Em termos teóricos o estudo contribui, pois avança na temática entre empresa familiar e desempenho empresarial, quando relaciona propriedade familiar com o desempenho econômico, financeiro e de mercado, bem como o nível ideal de propriedade para um desempenho favorável. A implicação prática consistiu em demonstrar que o desempenho das empresas familiares brasileiras, em comparação às empresas não familiares, nem sempre possui uma relação positiva com a propriedade. Os resultados alcançados poderão ser utilizados para aprimorar a gestão de negócios familiares, esses que no mundo possuem grande representatividade no desenvolvimento econômico.

Como qualquer pesquisa, essa também possui limitações. A primeira foi que estudo se limitou a comparar dados de empresas brasileiras com tailandesas. Desse modo, empresas familiares de outros países não foram consideradas. Como sugestões para pesquisas futuras, recomenda-se a ampliação considerando outros países, com cenários diferentes, bem como setores variados, para comparação dos resultados em outros contextos. Uma outra limitação refere-se a amostra, ao modelo e a técnica estatística aplicada, não podendo os resultados ser generalizados para todas as empresas familiares brasileiras. Como pesquisas futuras, sugere-se a aplicação de outros modelos e técnicas, em amostras nacionais diferentes, de forma a avançar as pesquisas entre propriedade familiar e desempenho empresarial.

\section{REFERÊNCIAS}

Abor, J. \& Biekpe, N. (2007). Corporate governance, ownership structure and performance of SMEs in Ghana: implications for financing opportunities. Corporate Governance, 7 (3), 288-300. DOI:10.1108/14720700710756562.

Acquaah, M. (2013). Management control systems, business strategy and performance: A comparative analysis of family and non-family businesses in a transition economy in sub-Saharan Africa. Journal of Family Business Strategy, 4 (2), 131-146. DOI:10.1016/j.jfbs.2013.03.002.

Altman, E. I. (1971). Railroad bankruptcy propensity. The Journal of Finance, 26 (2), 333-345. DOI:10.2307/2326049.

Anderson, R. C. \& Reeb, D. M. (2003). Founding-family ownership and firm performance:evidence from the S\&P 500. The Journal of Finance, 58 (3), 13011328. DOI:10.1111/1540-6261.00567.

Anderson, R. C., Duru, A. \& Reeb, D. M. (2012). Investment policy in family controlled firms. Journal of Banking \& Finance, 36 (61744-1758. DOI: 10.1016/j.jbankfin.2012.01.018.

Audretsch, D. B., Hülsbeck, M. \& Lehmann, E. E. (2013). Families as active monitors of firm performance. Journal of Family Business Strategy, 4 (2), 118-130. DOI: 10.1016/j.jfbs.2013.02.002. 
Andres, C. (2008). Large shareholders and firm performance - an empirical examination of founding-family ownership. Journal of Corporate Finance, 14 (1), 431-445. DOI:10.1016/j.jcorpfin.2008.05.003.

Barontini, R. \& Caprio, L. (2006). The effect of family control on firm value and performance: evidence from Continental Europe. European Financial Management, 12 (5), 689-723. DOI:10.1111/j.1468-036x.2006.00273.x.

Beaver, W. H. (1966). Financial ratios as predictors of failure. Journal of accounting research, 4 (1), 71-111. DOI:10.2307/2490171

Black, B. S. (1992). Institutional investors and corporate governance: the case for institutional voice. Journal of Applied Corporate Finance, 5 (3), 19-32. DOI:10.1111/j.1745-6622.1992.tb00223.x.

Casson, M. (1999). The economics of the family firm. Scandinavian Economics History Review, 47 (1), 10-23. DOI:10.1080/03585522.1999.10419802.

Colarossi, F., Giorgino, M., Steri, R. \& Viviani, D. (2008). A corporate governance study on Italian family firms. Corporate ownership \& control, 5 (4), 93-103. DOI:10.22495/cocv5i4p8.

Chen, C. R., Guo, W. \& Mande, V. (2003). Managerial ownership and firm valuation: evidence from Japanese firms. Pacific-Basin Finance Journal, 11 (3), 267-283. DOI:10.1016/s0927-538x(03)00024-6.

Chen, C. R. \& Steiner, T. L. (1999). Managerial ownership and agency conflicts: a nonlinear simultaneous equation analysis of managerial ownership, risk taking, debt policy, and dividend policy. The Financial Review, 34 (1), 119-136. DOI:10.1111/j.1540-6288.1999.tb00448.x.

Chung, K. H. \& Pruitt, S. W. (1994). A simple approximation of Tobin's Q. Financial Management, 23 (3), 70-74. DOI:10.2307/3665623

Demsetz, H. \& Villalonga, B. (2001). Ownership structure and corporate performance. Journal of Corporate Finance, 7 (3), 209-233. DOI:10.1016/s0929$1199(01) 00020-7$.

Filatotchev, I., Lien, Y. C. \& Piesse, J. (2005). Corporate governance and performance in publicly listed, family-controlled firms: evidence from Taiwan. Asian Pacific Journal of Management, 22, 257-283. DOI:10.1007/s10490-0053569-2.

Faccio, M., Lang, L. H. P. \& Young, L. (2001). Dividend and expropriation. American Economics Review, 91 (1), 54-78. DOI: 10.1257/aer.91.1.54

Family Firm Institute. (2017). Pontos de dados globais. Recuperado em: http://www.ffi.org/?page=GlobalDataPoints, 2017. Acesso em 12 setembro. 
Goes, T. H. M., Martins, H. H., \& Machado Filho, C. A. P. (2017). Desempenho Financeiro de Empresas com Características Familiares: Análise de Empresas Brasileiras Listadas na BM\&F. Revista de Gestão, 24(3), 197-209. DOI:10.1016/j.rege.2016.06.011.

Castro, G. R. \& Aguilera, R. V. (2014). Family involvement in business and financial performance: A set-theoretic cross-national inquiry. Journal of Family Business Strategy, 5 (1), 85-96. DOI:10.1016/j.jfbs.2014.01.006.

Holderness, C. G. \& Sheehan, D. P. (1988). The role of majority shareholders in publicly held corporations: an exploratory analysis. Journal of Financial Economics, 20, 317-346. DOI:10.1016/0304-405x(88)90049-9.

James, H. (1999). Owner as manager, extended horizons and the family firm. International Journal of Economics and Business, 6 (1), 41-56. DOI:10.1080/13571519984304.

Jensen, G.R., Solberg, D.P. \& Zorn, T.S. (1992). Simultaneous determination of insider ownership, debt and dividend policies. Journal of Financial and Quantitative Analysis, 27 (2), 247-263. DOI:10.2307/2331370.

Lunardi, M. A., Barbosa, E. T., Rodrigues Junior, M. M., Silva, T. P. \& Nakamura, W. T. (2017). Criação de Valor no Desempenho Econômico de Empresas Familiares e não Familiares Brasileiras. Revista Evidenciação Contábil \& Finanças, 5 (1), 94-112. DOI:10.18405/recfin20170106.

Martínez, J. I., Stöhr, B. S. \& Quiroga, B. F. (2007). Family ownership and firm performance: Evidence from public companies in Chile. Family Business Review, 20 (2), 83-95. DOI:10.1111/j.1741-6248.2007.00087.x.

McConnell, J. \& Servaes, H. (1990). Additional evidence on equity ownership and corporatevalue. Journal of Financial Economics, 27 (2), 595-612. DOI: 10.1016/0304-405X(90)90069-C

Minichilli, A., Brogi, M. \& Calabrò, A. (2015). Weathering the storm: Family ownership, governance, and performance through the financial and economic crisis. Corporate Governance (Oxford), 24 (6), 552-568. DOI:10.1111/corg.12125.

Morck, R. K., Shleifer, A. \& Vishny, R. (1988). Management ownership and market valuation: empirical analysis. Journal of Financial Economics, 20, 293-315. DOI: 10.1016/0304-405x(88)90048-7.

Oro, I. M., Beuren, I. M. \& Hein, N. (2009). Análise da eficiência de empresas familiares brasileiras. RAE-eletrônica, 8 (2), 1-27.

Oro, I. M., Beuren, I. M. \& Hein, N. (2009a). Análise da relação entre a estrutura de capital e o lucro operacional nas diversas gerações de empresas familiares brasileiras. Contabilidade Vista \& Revista, 20 (1), 67-94. 
Pound, J. (1991). Proxy voting and the SEC: investor protection versus market efficiency. Journal of Financial Economics, 29 (2), 241-285. DOl:10.1016/0304405x(91)90003-3.

Price Waterhouse Coopers. Pesquisa global sobre empresas familiares. Disponível em: $\quad$ http://www.pwc.com.br/pt/setores-de-atividade/empresasfamiliares/2017/tl_pgef_17.pdf. Acesso em: 26 junho, 2017.

Ross, S. A. (1977). The determination of financial structure: the incentive-signalling approach. Bell Journal of Economics, 8 (1), 23-40. DOI:10.2307/3003485.

Scarpin, J. E., Almeida, D. M. \& Machado, D. G. (2012). Endividamento e lucratividade: um estudo em empresas familiares e não familiares que compõem o índice IBRX-100 da B3. Revista Ambiente Contábil, 4 (2), 93-109.

Segura, L. C. \& Formigoni, H. (2014). Influência do controle e da gestão familiar no endividamento das empresas abertas Brasileiras: um estudo quantitativo. Brazilian Business Review, 11 (6), 51-76. DOI:10.15728/bbr.2014.1 1.6.3.

Shyu, J. (2011). Family ownership and firm performance: evidence from Taiwanese firms. International Journal of Managerial Finance, 7 (4), 397-411. DOI: $10.1108 / 17439131111166393$

Singh, M. \& Davidson, W.N. (2003). Agency costs, ownership structure and corporate governance mechanisms. Journal of Banking and Finance, 27, 793816. DOI:10.1016/s0378-4266(01)00260-6.

Trasobares, A. H. \& Górriz, C.G. (2016). Diversification and family control as determinants of performance: A study of listed business groups. European Research on Management and Business Economics, 3 (1), 46-54. DOI:10.1016/j.iedeen.2016.04.001.

Tres, G., Serra, F. \& Ferreira, M. (2014). O tempo de mandato do CEO e o desempenho das empresas: Um estudo comparativo de empresas familiares e não-familiares brasileiras. Revista Gestão \& Tecnologia, 14 (3), 5-31. DOI: 10.20397/2177-6652/2014.v14i3.732

Wang, K. T. \& Shailer, G. (2017). Family ownership and financial performance relations in emerging markets. International Review of Economics and Finance, 51, 82-98. DOI:10.1016/j.iref.2017.05.014.

Wooldridge, J. M. (2011). Introdução à Econometria: Uma Abordagem Moderna. Tradução da 4a. Edição Norte-Americana. 
Propriedade Familiar e Desempenho Empresarial: Evidências de Empresas Brasileiras que Compõem o Índice IBRX100 da B3

\section{CONTRIBUIÇÕES DOS AUTORES}

\begin{tabular}{|l|c|c|}
\hline \multicolumn{1}{|c|}{ Contribuição } & $\begin{array}{c}\text { Dalci Mendes } \\
\text { Almeida }\end{array}$ & Leonardo Flach \\
\hline 1. Idealização e concepção do assunto e tema da pesquisa & $\checkmark$ & $\checkmark$ \\
\hline 2. Definição do problema de pesquisa & $\checkmark$ & $\checkmark$ \\
\hline 3. Desenvolvimento da Plataforma Teórica & $\checkmark$ & \\
\hline 4. Delineamento da abordagem metodológica da pesquisa & $\checkmark$ & $\checkmark$ \\
\hline 5. Coleta de dados & $\checkmark$ & \\
\hline 6. Análises e interpretações dos dados coletados & $\checkmark$ & \\
\hline 7. Conclusões da pesquisa & $\checkmark$ & $\checkmark$ \\
\hline 8. Revisão crítica do manuscrito & $\checkmark$ & $\checkmark$ \\
\hline $\begin{array}{l}\text { 9. Redação final do manuscrito, conforme as normas } \\
\text { estabelecidas pela Revista. }\end{array}$ & $\checkmark$ & $\checkmark$ \\
\hline 10. Orientação & & $\checkmark$ \\
\hline
\end{tabular}

